\title{
Misdiagnosed and mismanaged atypical spinal tuberculosis: A case series report
}

\author{
FEIFEI PU* ${ }^{*}$, JING FENG $^{*}$, LIN YANG, LIN ZHANG and PING XIA \\ Department of Orthopedics, Wuhan No. 1 Hospital, Wuhan Integrated TCM \& Western Medicine Hospital, \\ Tongji Medical College, Huazhong University of Science and Technology, Wuhan, Hubei 430022, P.R. China
}

Received June 6, 2018; Accepted January 18, 2019

DOI: $10.3892 /$ etm.2019.8014

\begin{abstract}
Compared to other diseases, early or atypical spinal tuberculosis (TB) is prone to being misdiagnosed due to the lack of specific clinical manifestations and misleading negative results of various laboratory analyses and imaging examinations. Various methods for diagnosing spinal TB have become research hotspots. Recently, it has been proposed that effective vaccination is a mainstay of long-term policies to combat and control the TB epidemic. However, some new TB vaccines require further evaluation and clinical trials to demonstrate their efficiency and safety. Thus, early diagnosis and effective treatment are the other essential long-term strategies for controlling the TB epidemic immunization is closely associated with the diagnosis of spinal TB, which will become the focus of future research and set the direction for future developments. The present study provides a case series and a literature review of the characteristics of spinal TB to provide guidance for the diagnosis, treatment and prognosis of spinal TB.
\end{abstract}

\section{Introduction}

Spinal tuberculosis (TB) was first reported by Sternbach (1) in 1779, and it was named as Pott disease. Spinal TB is one of the most common extrapulmonary forms of TB, accounting for $\sim 1 \%$ of total TB cases and $50-60 \%$ of cases of osteoarticular TB (2). The early clinical manifestations of spinal TB are insidious, usually manifesting first as back pain and local tenderness, and with the subsequent progression of the disease, posterior kyphosis and nerve compression symptoms may develop.

Correspondence to: Professor Ping Xia, Department of Orthopedics, Wuhan No. 1 Hospital, Wuhan Integrated TCM \& Western Medicine Hospital, Tongji Medical College, Huazhong University of Science and Technology, 215 Zhongshan Road, Wuhan, Hubei 430022, P.R. China

E-mail: xiapingfm@126.com

*Contributed equally

Key words: spine, tuberculosis, atypical, misdiagnosis
Chemotherapy is an important part of the treatment of spinal $\mathrm{TB}$, and is an effective means of eliminating spinal lesions and prevent recurrence. When kyphotic deformity, neurological deficits or a huge abscess occurs, surgery becomes an efficient treatment option. Surgery is effective in eradicating TB foci, relieving spinal cord compression, re-establishing spinal stability and correcting the deformity (3).

According to a clinical epidemiological survey, the incidence of spinal TB is the highest in developing countries, and it has also been reported to be increasingly prevalent among immigrants in western countries (4). However, as compared to other diseases, early or atypical spinal TB is prone to being misdiagnosed due to the lack of specific clinical manifestations, and misleading negative results of various laboratory analyses and imaging examinations. Thus, atypical spinal TB is thought to be a diagnostic dilemma for surgeons. Atypical spinal TB has frequently been reported as case reports or case series (5); the available literature provides insufficient evidence and is not comprehensive enough to guide its treatment, particularly regarding the selection of surgical options, which may lead to inappropriate treatment.

From 2015 to 2018, 10 patients with atypical spinal TB were treated by surgery at our department. The present study summarizes the radiographic and clinical characteristics, and the therapeutic outcomes of these atypical spinal TB patients.

\section{Materials and methods}

The present study was approved by the Ethics Committee of the Wuhan No. 1 Hospital, Wuhan Integrated TCM \& Western Medicine Hospital (Wuhan, China). Written informed consent was obtained from all patients. From March 2015 to March 2018, 118 patients with spinal TB were diagnosed and treated by surgery at the Department of Orthopedics of Wuhan No. 1 Hospital, Wuhan Integrated TCM \& Western Medicine Hospital (Wuhan, China). Of these patients, 10 exhibited lesion involvement with an atypical radiographic presentation. Histopathological examinations and/or bacteriological cultures were used for the final diagnoses (1).

Patients were diagnosed using and X-ray machine. The test sites were imaged manually or by Automatic Exposure Control. Conventional plain CT was performed using dual-source CT, the instrument voltage was adjusted to $120 \mathrm{kV}$, the current was $200 \mathrm{~mA}$, the layer thickness was 2-3 $\mathrm{mm}$ and the layer 
spacing was $5 \mathrm{~mm}$. The images were processed by multiple planner reconstruction and surface shaded display in the post-processing station of spiral CT, and the vertebral body and spinal canal lesions were displayed. General MRI scanning was performed using 3T superconducting whole-body MRI scanners, and the scanning sequences included transverse T2WI, sagittal T2WI, sagittal T1WI and fat-suppressed T2WI. Transverse, coronal and sagittal axial helical scans were performed, and sagittal and axial enhanced scans were performed when necessary.

In all of the 10 patients of the present study, the diagnoses were confirmed by histopathological examinations and/or bacteriological cultures. Bone tissue or abscess samples were stained for acid-fast bacilli, mycobacterial organisms, in isolated cultures. Pus or bone tissues $(1 \times 1 \times 1 \mathrm{~cm})$ and necrotic intervertebral discs $(2 \mathrm{ml})$, and $2 \mathrm{ml}$ SDS-NaOH were added to the sterile centrifuge tube, which was agitated by a vortex shaker for a few sec. Then, the sample was agitated by a shaker at room temperature for $20 \mathrm{~min}$ and $50 \mathrm{ml}$ PBS was added. After centrifugation for $20 \mathrm{~min}$ at 3,000 x g at room temperature, a suspension was prepared with PBS and $0.5 \mathrm{ml}$ of the centrifugal precipitate in a sterile syringe. The pellets of the centrifugal precipitate were applied to the slide evenly, and the Ziehl-Neelsen acid fast staining was performed according to a previously described standard protocol (6). Dedicated liquid medium containing the specimens was incubated in a BACT/ALERT 3D system (cat. no. BTA3D; BioMérieux SA) at $37^{\circ} \mathrm{C}$ for 40 days. During the growth of mycobacterium tuberculosis, the metabolism of $\mathrm{CO}_{2}$ resulted in a change in $\mathrm{pH}$, which caused the color of the sensor to change from green to yellow, and the color sensor in the BACT/ALERT 3D system reported the results automatically and continuously.

CT- or ultrasonography-guided needle biopsies, or surgical biopsies were used to confirm the diagnosis. As a standard treatment, anti-TB drugs were administered for 9-12 months post-operatively and at all follow-ups until 36 months after the operation.

\section{Results}

Characteristics of patients. In the present study, 6 males and 4 females aged 8-56 years were included, and the median age was 34 years. The cohort included 3 cases with thoracic vertebra lesions, 1 with a thoracolumbar vertebra lesion, 5 with lumbar spine lesions and 1 with a sacral vertebra lesion. Medical records from 1-14 months previously were available and the average time-point was 3 months previously. Two patients were not diagnosed, whereas 8 patients were misdiagnosed. Among these misdiagnosed patients, 2 were diagnosed with lumbar muscle fasciitis, 3 with spinal tumors, 1 with intercostal neuralgia, 2 with osteoporotic fractures and 2 with lumbar disc herniation. Of the 10 patients, two had a history of chest TB and the remaining eight reported not to have been previously diagnosed with TB. In the present study, all of the patients had local pain and 2 cases $(20 \%)$ had a TB toxin reaction, including hypothermia, night sweats and weakness. Lower limb pain or numbness was present in 5 patients $(50 \%)$ and 6 cases $(60 \%)$ had a spinal activity limitation.
Laboratory test results. The erythrocyte sedimentation rate (ESR) was normal in 6 patients, while it ranged between $25-40 \mathrm{~mm} / \mathrm{h}$ in 4 patients, and it was clearly higher than the normal reference value (adult males, $0-10 \mathrm{~mm} / \mathrm{h}$; adult females, 0-12 mm/h) (2). Polymerase chain reaction (PCR) analyses of Mycobacterium tuberculi DNA content in peripheral blood may also be used to monitor TB treatment. These examinations are more specific than the tuberculin skin test (TST) for the diagnosis of TB infection, and their sensitivity varies. Among those individuals with low immunity, the T-SPOT is more sensitive than the TST. Although these tests detect TB, it is not possible to distinguish between an occult and active TB infection, and a further clinical assessment of the extent or activity of the disease is required if the T-SPOT/TST test is positive. Of the 10 patients, 3 patients had a normal blood sedimentation $(25-122 \mathrm{~mm} / \mathrm{h})$ and 3 patients had a positive tuberculin purified protein derivative test result. The ESR, PCR, TST and T-SPOT tests were performed by the clinical laboratory department of Wuhan No. 1 Hospital, Wuhan Integrated TCM \& Western Medicine Hospital.

Imaging examination results. In terms of diagnosis, typical cases are easy to diagnose, but the 10 cases with atypical spinal TB in the present study were difficult to diagnose as they has few symptoms and those they did exhibit were slight. Therefore, the X-ray could not sufficiently reveal their pathological features. In the present study, X-ray images exhibiting various degrees of bone destruction, narrowing of the intervertebral space, bone necrosis or shadows of cold abscesses were present in seven cases $(70 \%)$. A further eight cases $(80 \%)$ had positive signs on CT or MRI examinations (Table I). No recurrence in TB infections or lesions was identified, and all of the 10 patients presented with satisfactory clinical and radiological results.

Case report. A specific case of misdiagnosis is presented below. A 55 year-old female patient, whose chief complaint was lumbago for $>1$ year, presented with a 2 week history of limited motion. She was previously diagnosed with lupus erythematosus and chronic renal failure. The visual analogue scale (VAS) scores of the waist and lower limbs were 4 and 1, respectively. The sensory and motor functions were normal and no pathological sign was observed. Her ESR was $16 \mathrm{~mm} / \mathrm{h}$ and the C-reactive protein (CRP) level was $34.3 \mathrm{mg} / \mathrm{l}$. The T-SPOT test was negative. The MRI of the lumbar vertebral body exhibited patchy long T1 and T2 signals, and high short $\mathrm{T}$ inversion recovery (STIR) signals (Fig. 1). The patient was diagnosed with an osteoporotic compression fracture. Her back pain did not cease significantly even after one month; hence, she underwent a lumbar CT and MRI. The CT images revealed a small spot of lamellar low-density shadow and hardening of the edge around the L2 vertebral body. The MRI exhibited patchy long T1 and T2 signals and high STIR signals at the L1 and L2 vertebral bodies, but the disc was not invaded (Fig. 2). The patient denied any history of trauma and refused to undergo a biopsy. After 1 year the patient's waist pain became significantly worse and the VAS score were elevated to 6 . The patient's ESR was $71 \mathrm{~mm} / \mathrm{h}$ and the CRP level was $67 \mathrm{mg} / \mathrm{l}$. The T-SPOT test was weakly positive. An X-ray and 
Table I. Patient characteristics.

\begin{tabular}{|c|c|}
\hline Item & $\mathrm{N}$ \\
\hline \multicolumn{2}{|l|}{ Sex } \\
\hline \multicolumn{2}{|l|}{ Male } \\
\hline \multicolumn{2}{|l|}{ Female } \\
\hline \multicolumn{2}{|l|}{ Lesion location } \\
\hline \multicolumn{2}{|l|}{ Thoracic vertebra } \\
\hline \multicolumn{2}{|l|}{ Thoracolumbar vertebra } \\
\hline \multicolumn{2}{|l|}{ Lumbar vertebra } \\
\hline \multicolumn{2}{|l|}{ Sacral vertebra } \\
\hline \multicolumn{2}{|l|}{ Known history of chest TB } \\
\hline \multicolumn{2}{|l|}{ Yes } \\
\hline \multicolumn{2}{|l|}{ No } \\
\hline \multicolumn{2}{|l|}{ Clinical manifestations } \\
\hline \multicolumn{2}{|l|}{ Local pain } \\
\hline \multicolumn{2}{|l|}{ Tuberculous toxin reaction } \\
\hline \multicolumn{2}{|l|}{ Lower limb pain/numbness } \\
\hline \multicolumn{2}{|l|}{ Spinal activity limitation } \\
\hline \multicolumn{2}{|l|}{ Auxiliary examination } \\
\hline \multicolumn{2}{|l|}{$\operatorname{ESR}(+)$} \\
\hline \multicolumn{2}{|l|}{ PPD (+) } \\
\hline \multicolumn{2}{|l|}{ X-ray (+) } \\
\hline \multicolumn{2}{|l|}{ CT/MRI (+) } \\
\hline \multicolumn{2}{|l|}{ Initial diagnosis } \\
\hline \multicolumn{2}{|l|}{ Spinal TB } \\
\hline \multicolumn{2}{|l|}{ Lumbar muscle fasciitis } \\
\hline \multicolumn{2}{|l|}{ Spinal tumors } \\
\hline \multicolumn{2}{|l|}{ Spinal intercostal neuralgia } \\
\hline Osteoporotic fractures & 2 \\
\hline Lumbar disc herniation & \\
\hline
\end{tabular}

CT, computed tomography; MRI, magnetic resonance imaging; ESR, electron spin resonance; PPD, tuberculin purified protein derivative test; TB, tuberculosis.

CT revealed vertebral bone destruction, narrowing of the intervertebral space and partial necrosis (Fig. 3). The MRI exhibited patchy long T1 and T2 signals and high STIR signals at the L1 and L2 vertebral bodies; these indicated heterogeneous signal changes in multiple vertebrae and a paravertebral abscess with intervertebral disc involvement (Figs. 3 and 4). Anti-TB drugs were prescribed for 2 weeks although the physical examination revealed no obvious positive indicators. The patient was subjected to excision of anterior L2-3 foci, abscess debridement and autograft fusion with instrumentation. The diagnosis was confirmed by bacteriological cultures of the abscess. A post-operative pathological section exhibited large areas of inflammatory granulation tissue, dense fibrous connective tissue and cartilage, and a small number of dead bone fragments (Fig. 5). During the post-operative follow-ups, no abscess was detected. Thus far, the patient has continued with chemotherapy and no recurrence was detected during the 15 month follow-up.
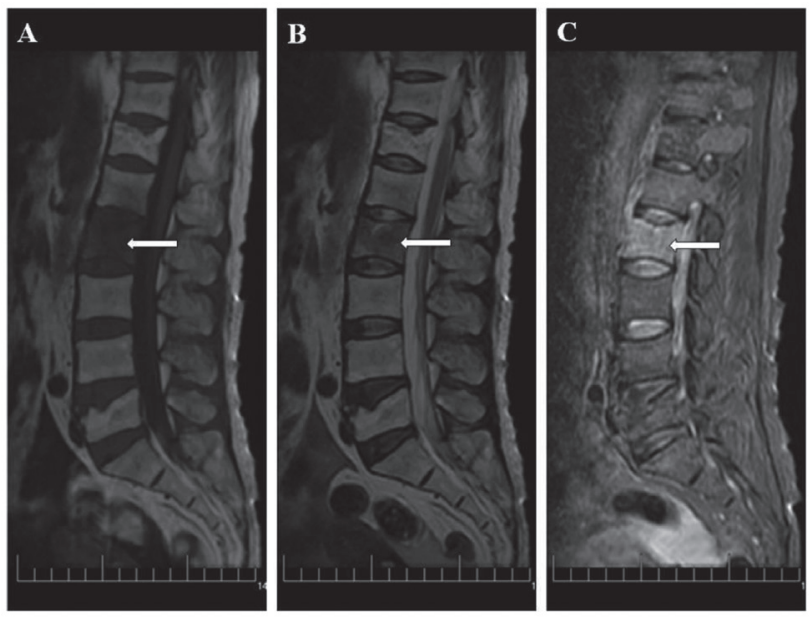

Figure 1. Magnetic resonance images of the lumbar vertebral body. The patient was a 54-year-old female patient, who underwent magnetic resonance imaging (visual field, 40x40x40 cm; layer thickness, $5 \mathrm{~mm}$; layer spacing, $3 \mathrm{~mm} ; \mathrm{b}, 800 \mathrm{sec} / \mathrm{mm}^{2}$ ) in November 2016 due to lower back pain. The patient had not undergone any other tests or treatment. (A) Long T1 signal in the sagittal plane. (B) Long T2 signal in the sagittal plane. (C) High short $\mathrm{T}$ inversion recovery signal in the sagittal plane. Small grid, $1 \mathrm{~cm}$. The lesion is indicated by white arrows.

\section{Discussion}

Early clinical manifestations of spinal TB are atypical and insidious. The earliest signs are stiffness in the upper back with muscle contraction; this may be due to the lesion area developing tension to produce the corresponding spine-chest-neck stiffness. Spinal rigidity is not a specific manifestation for the diagnosis of the disease; however, it provides a powerful clue. It is important to note that these clinical manifestations are also common in other types of spinal disease; therefore, it is necessary to inquire about the patients' history and confirm whether a history of a pulmonary disease or TB is present.

The causes of pain in the spine from the cervical segment to the sacral segment are complex; back pain is a common and the earliest clinical symptom of a spinal tumor. In addition, a spinal tumor and spinal TB may occur as a result of mild to severe progression of spinal cord dysfunction, including nerve root pain, sensorimotor dysfunction, urinary incontinence and even paraplegia. Bone destruction may result in pathologies including vertebral body collapse, cuneate and spinal deformity; in fact, certain spinal tumors may produce similar bone destruction and spinal deformity (7).

Radiographic examination is of great diagnostic value, and the relevant pathological features include vertebral space stenosis and paravertebral shadow, as well as vertebral body destruction and compression. In terms of diagnosis, typical cases are easy to diagnose, spinal TB has typical imaging characteristics, including adjacent vertebral body bone destruction, intervertebral disc destruction and vertebral abscess formation. In general, spinal TB results in extensive destruction of intervertebral disc and then the vertebral body; however, the narrowing or disappearance of the intervertebral space is associated with this type of lesion. Central lesions of spinal TB affect the perivertebral body at a late stage. In elderly patients, spinal TB generally does not result in paravertebral abscess or smaller lesions, which is associated with the weak response in those patients. 

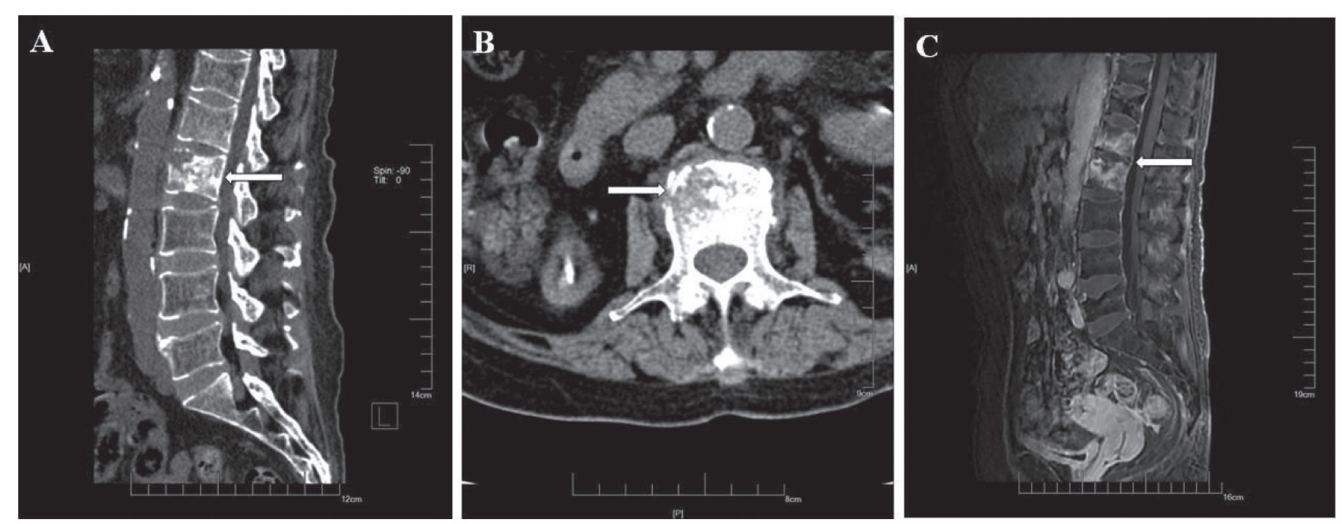

Figure 2. Computed tomography and magnetic resonance imaging images of the lumbar vertebral body in a 54-year-old female patient on December 30, 2016. The patient did not receive any treatment after the first examination. (A) On computed tomography, a small-spot lamellar low-density shadow and a hardening edge around the L2 vertebral body in radiographs in the sagittal plane were visible, with no significant compression in vertebral height. (B) In the transverse plane of the vertebral body, the destruction of the vertebrae did not encroach on the spinal canal, and there was no compression of the dura or spinal cord. (C) On magnetic resonance imaging (visual field, 40x40x40 cm; layer thickness, $5 \mathrm{~mm}$; layer spacing, $3 \mathrm{~mm}$; b, $800 \mathrm{sec} / \mathrm{mm}^{2}$ ), patchy long T1 and T2 signals, and a high short T inversion recovery signal of the L1 and L2 vertebral body in the sagittal plane were observed, without invasion of the disk. Small grid, $1 \mathrm{~cm}$. The lesion is indicated by white arrows.
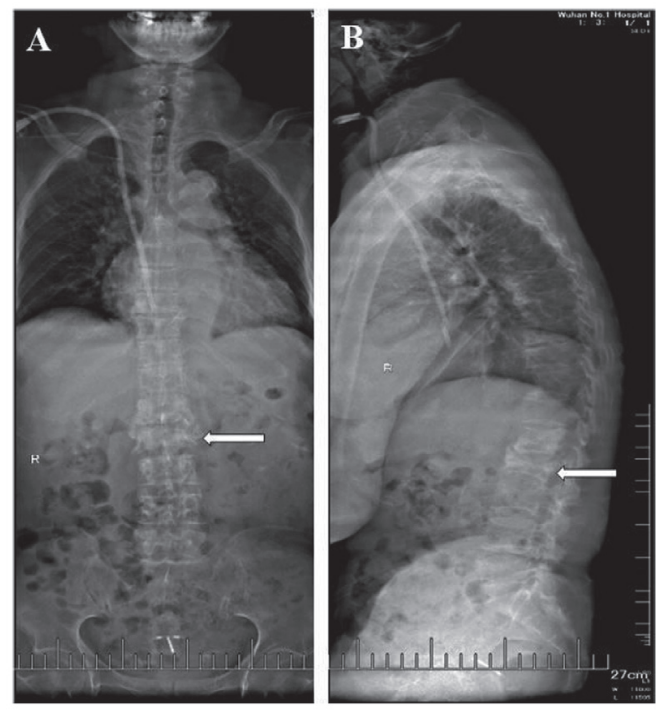

Figure 3. X-ray of the lumbar vertebral body in a 54-year-old female patient on June 26, 2017. The patient did not receive any treatment. (A) Anteroposterior $\mathrm{X}$-ray in the coronal plane and (B) lateral X-ray in the sagittal plane. Destruction of the vertebral bone, narrowing of the intervertebral space and partial necrosis was observed. Small grid, $1 \mathrm{~cm}$. The lesion is indicated by white arrows.

The gold standard for the traditional diagnosis is the examination of clinical samples of the patients, namely a blood smear test for Mycobacterium tuberculi, along with typical histological examinations and laboratory tests including complete blood count, ESR and CRP, as well as further auxiliary examinations to diagnose the corner curriculum $(8,9)$. However, due to the growth requirements and slow growth rate of TB bacilli, their culture is challenging. The TST is frequently performed, but its sensitivity and specificity are limited, even in high-incidence areas of TB; even after in individuals with repeated exposure to TB bacillus, a $\sim 20 \%$ negative rate of the TST has been estimated (8).

There are 3 types of non-cultivation laboratory examination methods to detect TB bacillus: i) Immunological detection including the tuberculin experiment (in vitro detection of the antibody against mycobacterium TB in the white blood cells) using ICT Tuberculosis (AMRAD-ICT; Sydney, Australia) to detect mycobacterium TB in whole blood. It allows for a rapid and accurate diagnosis of TB (10). ii) Detection of metabolites: a) In vitro interferon (IFN) test-when T-lymphocytes come into contact with mycobacterium, the lymph cells secrete a significant amount of IFN- $\gamma$; b) T-SPOT technology of ELISA (11); iii) PCR technology to amplify the DNA of the TB mycobacterium (including repeated sequencing of IS6110), and the results may be obtained within a few hours (12).

$\mathrm{X}$-ray imaging may roughly determine the location of the TB lesion and affected area of the spine, and it is conducive to the detection of spinal TB (13). The X-ray image may appear normal if the lesions are in the early stage, while progressive aggravation of the lesions may lead to intervertebral space stenosis following intervertebral disc involvement. Attention should be paid regarding changes in bone density, osteoporotic lesions, vertebral collapse and abnormalities in the physiological curvature of the spine. X-ray imaging may also fail to detect osteoporotic fractures and congenital diseases. It also has the following disadvantages: Slight bone changes and small calcification areas may not be easily identified and it is also difficult to diagnose cold abscesses by X-ray imaging.

A CT scan is able to detect even small bone damages at an early stage, even at specific sites, including the atlantoaxial and cervicothoracic junctions, which are difficult to be observed on X-ray imaging. A three-dimensional CT image may be used to assess the damage of the spine as a whole, indicating the location of TB, invasion into the spinal canal and the degree of spinal stenosis. The damage is mainly categorized into the debris type, osteolysis type and sub-periosteum type. However, the CT examination also has certain disadvantages: The original image does not reveal any abnormalities in the intervertebral space and it is difficult to visualize soft tissue lesions $(14,15)$.

Among all the imaging modalities, MRI is the best method to diagnose spinal TB, particularly for the early diagnosis. 

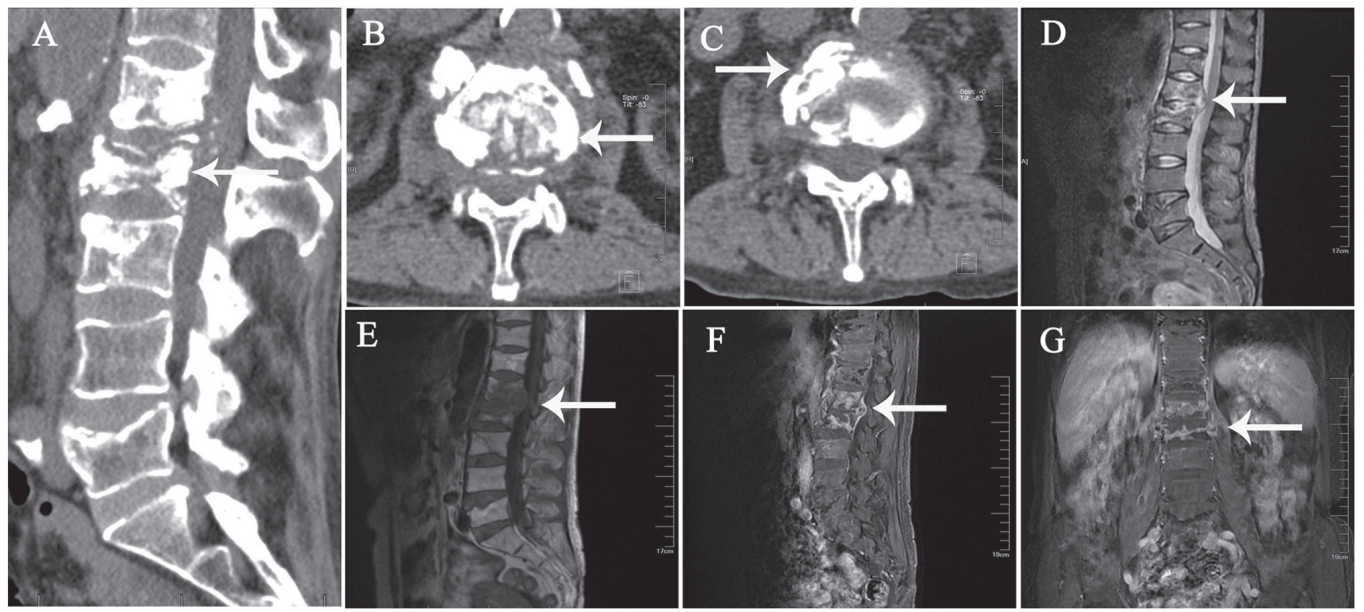

Figure 4. CT and MRI images of the lumbar vertebral body in a 54 year-old female patient on June 26, 2017. The patient did not receive any treatment after examination. (A) In the sagittal plane on CT, the destruction of the vertebral body and local bone sclerosis were apparent, and the vertebral body was highly compressed. (B) Cross-sectional view of the vertebral body revealing bone destruction and compression of local dura in the transverse plane. (C) CT cross-sectional view indicates partial necrosis of the vertebral body in the transverse plane. (D-G) MRI (visual field, $40 x 40 x 40 \mathrm{~cm}$; layer thickness, 5 mm; layer spacing, $3 \mathrm{~mm}$; b, $800 \mathrm{sec} / \mathrm{mm}^{2}$ ). (D) Patchy long T1 signal of the L1 and L2 vertebral body in the sagittal plane, (E) patchy long T2 signal of the L1 and L2 vertebral body in the sagittal plane and (F) high short T inversion recovery signal of the L1 and L2 vertebral body in the sagittal plane. Heterogeneous signal changes in multiple vertebrae and a paravertebral abscess, with intervertebral disc involvement were observed. (G) Contrast-enhanced MRI revealed intervertebral disc involvement in the coronal plane. Small grid, $1 \mathrm{~cm}$. The lesion is indicated by white arrows. CT, computed tomography; MRI, magnetic resonance imaging.

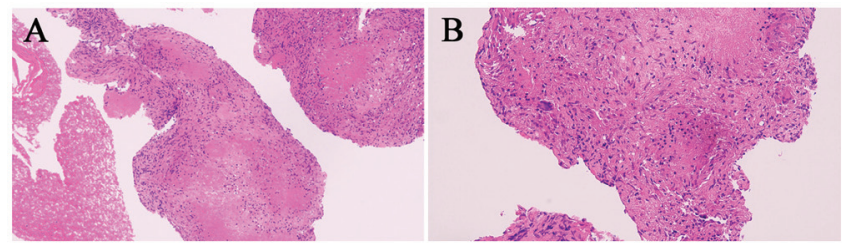

Figure 5. Post-operative pathological sections. (A) Large areas of inflammatory granulation tissue. Magnification x100. (B) Dense fibrous connective tissue, cartilage and a small number of dead bone fragments. Magnification x200.

When no abnormality is detected on X-ray imaging and even the CT image is not clear, the MRI screening does not only indicate the number and range of the involved vertebral segments clearly, but also reveals the condition of the paravertebral soft tissues $(16,17)$. The MRI characteristics of spinal TB are as follows: Lesions in 2 or more adjacent vertebral bodies are most frequent; vertebral body destruction originating from anterior and central segments may be present; T1-weighted imaging (T1WI) signals are low and T2WI signals are mixed; vertebral body damage may display as multiple necrosis; and the abscess area may vary in size. Obvious enhancement of cricoid attachment is involved in rare cases.

The MRI analysis of the representative cases in the present study revealed that the intervertebral disc was deformed and flattened with an unclear edge; abnormal signal intensities were recorded and the severely damaged intervertebral disc disappeared, leading to vertebral body fusion. The vertebral body around the vertebral disc corresponded to the bone damage and the shape of the vertebral body was incomplete, mostly wedge-shaped. However, if the vertebral body of the spine is free from intervertebral discitis and soft tissue signal abnormalities during the inflammation, it is difficult to distinguish spinal TB from a spinal tumor, and a biopsy may be performed to confirm the diagnosis.
B-ultrasonography has obvious advantages in spinal TB with paravertebral or lumbar abscess. The area of the abscess may be observed as a dark area of fluid and the dead bone area is strongly echogenic. The use of B-ultrasonography is simple, safe and fast. It may also be used to guide the local positioning of a puncture drainage, an indwelling drainage tube or the injection of therapeutic drugs (14).

The clinical features of early spinal TB are not typical and may be misdiagnosed as those of other diseases $(2,8)$, including the following: i) If one vertebral body or several discontinuous segments are involved during the imaging for spinal TB, this may easily be misdiagnosed as a spinal tumor; ii) intervertebral disc inflammation: The patient has a high fever, which is different from the afternoon low fever of TB patients. The imaging may display no damage to the cartilage endplate and no paravertebral cold abscess; iii) ankylosing spondylitis: $\mathrm{X}$-ray images of the spine showed vertebral osteoporosis, quadrate degeneration, facet joint fuzziness, calcification of the paravertebral ligaments, and the formation of bone bridges. 'Bamboo-like' changes in the vertebral column may be observed on X-ray imaging. However, there are certain further spinal diseases that require to be differentiated from spinal TB, including suppurative infection of the spine and eosinophilic granuloma. Hence, for the definitive diagnosis of spinal TB, comprehensive judgment of the latest detection methods such as AMRAD ICT, T-SPOT, PCR, spinal biopsy and sepsis bacteriology studies are required.

In conclusion, if spinal TB is diagnosed at an early stage, early treatment may be initiated. This does not only interfere with the progression of the disease and shorten the course of treatment, but also reduces the economic burden and the risk of the occurrence of spinal deformity. It is apparent that surgery is an important adjunct, but does not cure active TB. An early and more accurate diagnosis of spinal TB will become feasible with the continuous improvement of the examination methods. 


\section{Acknowledgements}

The authors would like to thank Dr Wei Liu and Dr Xiaolong Zhao from Department of Orthopedics, Wuhan No. 1 Hospital, Wuhan Integrated TCM \& Western Medicine Hospital, Tongji Medical College, Huazhong University of Science and Technology (Wuhan, China) for providing technical assistance.

\section{Funding}

This work was supported by grants from the Research Project of Hubei Province Health and Family Planning Commission (grant no. WJ2017Z022), the Natural Science Fund Project of Science and Technology Department of Hubei Province (grant no. 2016CFB666), a Special Clinical Research Fund of Wu Jieping Medical Foundation (grant no. 320.6750.17566), the Scientific Research Program of Wuhan Health and Family Planning Commission (grant nos. WX17Q38 and WZ18Q05) and the Research Program of Wuhan No. 1 Hospital, Wuhan Integrated TCM \& Western Medicine Hospital (grant no. 2017Y01).

\section{Availability of data and materials}

The datasets generated and/or analyzed during the current study are not publicly available due to patient privacy and copyright of case data, but are available from the corresponding author on reasonable request.

\section{Authors' contributions}

FP and JF conceived and designed the experiments of the current study. LY and LZ contributed to the admission and treatment of the patients. LY, LZ, FP and PX performed the collection and collation of data. PX analyzed and interpreted the data. All authors have written the manuscript, and read and approved the final manuscript.

\section{Ethics approval and consent to participate}

The present study was approved by the Ethics Committee of the Wuhan No. 1 Hospital, Wuhan Integrated TCM \& Western Medicine Hospital (Wuhan, China). Written informed consent was obtained from all patients.

\section{Patient consent for publication}

Consent for publication of images was obtained from all patients.

\section{Competing interests}

The authors declare that they have no competing interests regarding this study.

\section{References}

1. Sternbach G: Percivall Pott: Tuberculous spondylitis. J Emerg Med 14: 79-83, 1996.

2. Dunn RN and Ben Husien M: Spinal tuberculosis: Review of current managment. Bone Joint J 100: 425-431, 2018.

3. Varatharajah S, Charles YP, Buy X, Walter A and Steib JP: Update on the surgical management of pott's disease. Orthop Traumatol Surg Res 100: 229-235, 2014

4. Wang Y, Wang Q, Zhu R, Yang C, Chen Z, Bai Y, Li M and Zhai X: Trends of spinal tuberculosis research (1994-2015): A bibliometric study. Medicine (Baltimore) 95: e4923, 2016.

5. D'souza MM, Mondal A, Sharma R, Jaimini A and Khanna U: Tuberculosis the great mimicker: $18 \mathrm{~F}$-fludeoxyglucose positron emission tomography/computed tomography in a case of atypical spinal tuberculosis. Indian J Nucl Med 29: 99-101, 2014.

6. Marks J: Notes on the ziehl-neelsen staining of sputum. Tubercle 55: 241-244, 1974.

7. Garg RK and Somvanshi DS: Spinal tuberculosis: A review. J Spinal Cord Med 34: 440-454, 2011.

8. Chen $\mathrm{CH}$, Chen YM, Lee CW, Chang YJ, Cheng CY and Hung JK: Early diagnosis of spinal tuberculosis. J Formos Med Assoc 115: 825-836, 2016.

9. Merino P, Candel FJ, Gestoso I, Baos E and Picazo J: Microbiological diagnosis of spinal tuberculosis. Int Orthop 36: 233-238, 2012.

10. Delogu G, Zumbo A and Fadda G: Microbiological and immunological diagnosis of tuberculous spondylodiscitis. Eur Rev Med Pharmacol Sci 16: 73-78, 2012.

11. Yuan K, Zhong ZM,Zhang Q, Xu SC and Chen JT: Evaluation of an enzyme-linked immunospot assay for the immunodiagnosis of atypical spinal tuberculosis (atypical clinical presentation/atypical radiographic presentation) in China. Braz J Infect Dis 17: 529-537, 2013.

12. Sharma K, Meena RK, Aggarwal A and Chhabra R: Multiplex PCR as a novel method in the diagnosis of spinal tuberculosis-a pilot study. Acta Neurochir (Wien) 159: 503-507, 2017.

13. Zhang $\mathrm{H}$ and Lu Z: Atypical imaging of spinal tuberculosis: A case report and review of literature. Pan Afr Med J 24: 101, 2016.

14. Ansari S, Amanullah MF, Ahmad K and Rauniyar RK: Pott's spine: Diagnostic imaging modalities and technology advancements. N Am J Med Sci 5: 404-411, 2013.

15. Rivas-Garcia A, Sarria-Estrada S, Torrents-Odin C, Casas-Gomila L and Franquet E: Imaging findings of pott's disease. Eur Spine J 22: 567-578, 2013.

16. Kilborn T, Janse van Rensburg P and Candy S: Pediatric and adult spinal tuberculosis: Imaging and pathophysiology. Neuroimaging Clin N Am 25: 209-231, 2015.

17. Kumar Y, Gupta N, Chhabra A, Fukuda T, Soni N and Hayashi D: Magnetic resonance imaging of bacterial and tuberculous spondylodiscitis with associated complications and non-infectious spinal pathology mimicking infections: A pictorial review. BMC Musculoskelet Disord 18: 244, 2017.

This work is licensed under a Creative Commons Attribution-NonCommercial-NoDerivatives 4.0 International (CC BY-NC-ND 4.0) License. 